\title{
DISCRIMINAÇÃO DE LINHAGENS DE MILHO QUANTO À UTILIZAÇÃO DE NITROGÊNIO, POR MEIO DA AVALIAÇÃO DE CARACTERISTICAS DO SISTEMA RADICULAR
}

\author{
MARCELO OLIVEIRA SOARES ${ }^{1}$, IVANILDO EVÓDIO MARRIEL ${ }^{2}$, PAULO CÉSAR \\ MAGALHÃES ${ }^{2}$, LAURO JOSÉ MOREIRA GUIMARÃES ${ }^{2}$, FERNANDO RODRIGO DE \\ OLIVEIRA CANTÃO ${ }^{3}$, MICHEL CASTELLANI DA ROCHA ${ }^{4}$, GERALDO AFONSO DE \\ CARVALHO JÚNIOR ${ }^{5}$ GLAUCO VIEIRA MIRANDA ${ }^{6}$
}

\begin{abstract}
${ }^{1}$ Doutorando em Genética e Melhoramento - Universidade Federal de Viçosa, UFV, email: marcelosoares2001@gmail.com CEP 36570-000

${ }^{2}$ Pesquisadores, Drs. Embrapa Milho e Sorgo Caixa Postal 151, CEP 35701-970, Sete Lagoas, MG, ${ }^{3}$ Engenheiro Agrônomo, Msc Fisiologia Vegetal, University of Illinois at Urbana-Champaign - UIUC ${ }^{4}$ Bioquímico, Msc Fitotecnia - Universidade Federal de Viçosa - UFV

${ }^{5}$ Estudante de Graduação - Universidade Federal de Viçosa - UFV

${ }^{6}$ Professor Universidade Federal de Viçosa, Departamento de Fitotecnia, email: glaucovmiranda@ufv.br, CEP36570-000
\end{abstract}

Revista Brasileira de Milho e Sorgo, v.8 n.1, p. 93-103, 2009

RESUMO - O objetivo deste trabalho foi discriminar linhagens endogâmicas de milho por meio da avaliação de características radiculares fornecidas pela ánalise de imagens digitais, em doses contrastantes de nitrogênio no solo. Foram avaliadas seis linhagens endogâmicas de milho pertencentes à Embrapa Milho e Sorgo, em vasos de cinco litros contendo Latossolo Vermelho, textura média, em dois níveis diferenciados de adubação nitrogenada (zero e $2 \mathrm{mmol} . \mathrm{L}^{-1}$ ), na forma de nitrato de amônio. O delineamento experimental utilizado foi o de blocos casualizados, em esquema fatorial, com três repetições. Foi realizada a avaliação de características de raiz e parte aérea das plantas colhidas aos 23 dias após a semeadura. Foram observadas interações significativas entre as linhagens e doses de nitrogênio para comprimento radicular, superfície radicular, comprimento de raízes muito finas, superfície de raízes muito finas e superfície de raízes grossas, volume de raízes muito finas, massa seca da parte aérea e massa seca total. A linhagem CMS 28-7.1 apresentou menor proporção do volume da raiz muito fina/volume radicular em relação às outras linhagens. Em baixa disponibilidade de nitrogênio, as características radiculares diferenciaram as linhagens de milho e o volume de raízes muito finas.

Palavras-chave: Zea mays, estresse de nitrogênio, morfologia de raiz, WinRhizo 


\title{
DISCRIMINATION OF MAIZE LINES AS TO NITROGEN UTILIZATION THROUGH EVALUATION OF ROOT SYSTEM CHARACTERISTICS
}

\begin{abstract}
The objective of this study was to discriminate maize inbred lines by evaluating root characteristics obtained by digital images analysis when different nitrogen (N) rates were applied. Six maize inbred lines from Embrapa Maize and Sorghum were grown in five liter pots containing Red Latosol - medium textured, and two different $\mathrm{N}$ rates (zero and 2 mmol. $\mathrm{L}^{-1}$ ) were used. Nitrogen was applied as ammonium nitrate. A randomized complete blocks design was used, with three replications. Root and shoot features were evaluated on plants harvested 23 days after sowing. Significant interactions were observed between strains and $\mathrm{N}$ rates for root length, root surface area, very fine root length, surface area of very fine roots, surface area of thick roots, very fine root volume, shoot dry matter and total dry matter. The inbred line CMS 28-7.1 showed a lower very fine root volume/total root volume ratio compared to the other inbred lines. At low $\mathrm{N}$ availability, root characteristics allowed to distinguish maize inbred lines and very fine roots volume.
\end{abstract}

Key words: Zea mays, nitrogen stress, root morphology, WinRhizo

O nitrogênio é o nutriente mais utilizado pela planta de milho, estando relacionado com a produtividade final de grãos. Assim, o estresse causado pela baixa disponibilidade de nitrogênio é um dos mais severos, levando a uma drástica redução da produtividade (Banziger et al., 2000).

$\mathrm{O}$ uso de fertilizantes nitrogenados em excesso pode, também, provocar prejuízos ambientais, como a contaminação do lençol freático com nitrato, devido à eficiência da adubação nitrogenada ser por volta de apenas 50\% (Gallais \& Hirel, 2004; Deuner et al., 2008; Hodgen et al., 2009; Roberts et al., 2009).

A avaliação de genótipos de milho em condições de baixa disponibilidade de $\mathrm{N}$ tem mostrado variabilidade genética no germoplasma tropical (Miranda et al., 2005; Fidelis et al., 2007; Souza et al., 2008). Por outro lado, essa seleção pode reduzir a produtividade de grãos (Presterl et al., 2003) e, portanto, somente deve ser realizada quando a redução na produtividade não exceda $40 \%$ em relação à alta disponibilidade de N (Gallais \& Coque, 2005).

A seleção de plantas de milho para eficiência na utilização de nitrogênio pode ser realizada por meio de características secundárias, como as relacionadas ao sistema radicular. A característica secundária ideal é aquela que esteja correlacionada geneticamente com a produtividade de grãos em estresse, 
que apresente alta herdabilidade, possua alta variabilidade genética, seja fácil e rápida de avaliar, estável durante o período de medição, seja observada antes do florescimento, para que genitores indesejados não sejam cruzados, e seja um estimador real da produção de grãos antes da colheita final (Edmeades et al., 1998; Bänziger et al., 2002; O’Neill et al., 2006).

Uma técnica usada para medir o sistema radicular é a avaliação de parâmetros radiculares oriundos da análise de imagens, baseada no escaneamento digital de raízes, sendo utilizada por Rubio \& Lynch (2007), para medir comprimento de raízes de feijão (Phaseolus vulgaris $L$ ) em alta e baixa disponibilidade de fósforo, em solução nutritiva.

Assim, este trabalho teve como objetivo discriminar linhagens endogâmicas de milho, por meio da avaliação de características radiculares, fornecidas pela análise de imagens digitais, em doses contrastantes de nitrogênio no solo.

O experimento foi realizado na Embrapa Milhoe Sorgo, em Sete Lagoas, MG, em condição de casa-de-vegetação. Foram semeadas cinco sementes por vaso (com volume de cinco $\mathrm{dm}^{-3}$ de solo) das linhagens endogâmicas de milho CMS 28-19.1 e CMS 28-9.2, além das linhagens pré-classificadas como eficientes (CMS 28-7.1 e CMS 28-8.1) e ineficientes (CMS 28-10.2 e CMS 28-17.2) no uso de nitrogênio, segundo Purcino et al. (2008). Foi utilizado o delineamento em blocos ao acaso, em esquema fatorial $6 \times 2$, sendo seis linhagens e duas doses de nitrogênio, com três repetições. As doses constituíram da não aplicação de nitrogênio $(\mathrm{N}-)$ e da aplicação de $2 \mathrm{mmol} \mathrm{L}^{-1}$ de $\mathrm{N}(\mathrm{N}+)$, realizada aos 10, 15 e 18 dias após a semeadura (DAS), usando o nitrato de amônio como fonte de fertilizante nitrogenado.

A adubação com os outros nutrientes foi realizada com base na análise de solo, a qual apresentou as seguintes características: $\mathrm{pH}$ 6,4;

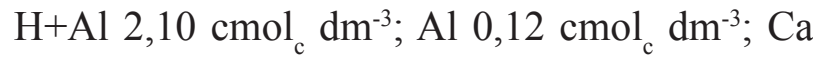

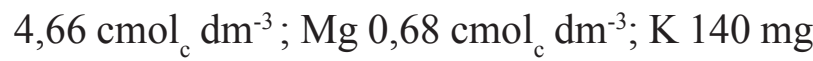
$\mathrm{dm}^{-3}$; P $34 \mathrm{mg} \mathrm{dm}^{-3}$; matéria orgânica 2,13 dag $\mathrm{kg}^{-1}$; soma de bases 5,69 $\mathrm{cmol}_{\mathrm{c}} \mathrm{dm}^{-3}$; CTC 7,97 $\mathrm{cmol}_{\mathrm{c}} \mathrm{dm}^{-3}$; V(Saturação por base) $73 \%$.

Duas plantas por vaso foram colhidas com 23 DAS, o solo foi destorroado e, após o sistema radicular ter sido lavado, este foi armazenado em frascos contendo solução de etanol 70\%, a fim de evitar a desidratação dos tecidos, para posterior avaliação de sua morfologia.

As características radiculares foram obtidas por meio da análise de imagens do software WinRhizo v. 4.0, Regent Systems, Quebec, (Canadá), acoplado a um scanner, utilizando definição de 400 dpi para as medidas de morfologia de raiz (Bouma et al., 2000). As leituras foram realizadas em duas plantas por linhagem, sendo que, para o comprimento, superfície e volume, as raízes foram classificadas em classes de diâmetro, conforme descrito por Bhom (1979) e modificado por Cantão (2007), 
sendo as raízes agrupadas nas classes a seguir: muito finas $(\varnothing<0,5 \mathrm{~mm})$, finas $(0,5 \mathrm{~mm}<\varnothing<2$ $\mathrm{mm})$ e grossas $(\varnothing>2 \mathrm{~mm})$.

Assim, foram avaliados comprimento radicular $(\mathrm{CR}, \mathrm{cm})$, superfície radicular (SR, $\mathrm{cm}^{2}$ ), volume radicular (VR, $\mathrm{cm}^{3}$ ), comprimento de raízes muito finas (CRMF, $\mathrm{cm}$ ), comprimento de raízes finas $(\mathrm{CRF}, \mathrm{cm})$, comprimento de raízes grossas (CRG, $\mathrm{cm})$, superfície de raízes muito finas $\left(\mathrm{SRMF}, \mathrm{cm}^{2}\right)$, superfície de raízes finas $\left(\mathrm{SRF}, \mathrm{cm}^{2}\right)$, superfície de raízes grossas $(\mathrm{SRG}$, $\mathrm{cm}^{2}$ ), volume de raízes muito finas (VRMF, $\left.\mathrm{cm}^{3}\right)$, volume de raízes finas (VRF, $\mathrm{cm}^{3}$ ) e volume de raízes grossas $\left(\mathrm{VRG}, \mathrm{cm}^{3}\right)$.

Após a obtenção dessas características, as raízes e a parte aérea da planta foram secadas em estufa, com ventilação forçada a $72^{\circ} \mathrm{C}$, até a obtenção de massa constante e, então, pesadas, para a determinação da massa seca de raiz (MSR), em gramas/vaso, e da massa seca de parte aérea (MSPA), em gramas/vaso. Foram, também, calculadas a massa seca total (MSTO) e a relação MSPA/MSR.

Procedeu-se às análises de variância e à separação das médias, pelo teste de Tukey, utilizando-se o Programa de análises genéticas GENES (Cruz, 2001).

O comprimento e a superfície radicular, o comprimento de raízes muito finas, a superfície de raízes muito finas, a superfície de raízes grossas, o volume de raízes muito finas, o volume de raízes grossas, a massa seca da parte aérea e a massa seca total das linhagens de milho apresentaram interação significativa com as doses de nitrogênio. Assim, essas características diferenciaram as linhagens em alta e baixa disponibilidade de nitrogênio, mostrando a relação entre o sistema radicular e a disponibilidade do nutriente (Tabela 1). Interações significativas entre híbridos de milho e doses crescentes de nitrogênio, por meio da avaliação do comprimento e superfície radicular, também foram encontradas por Costa et al. (2002), em estudo realizado em casa-devegetação.

A média geral da massa seca da parte aérea (MSPA) e da massa seca total (MSTO) das seis linhagens diminuiu em face de baixa disponibilidade de $\mathrm{N}$ no solo. Embora não tenha havido diferença significativa na média geral da MSR, entre as duas doses de nitrogênio, a média geral das seis linhagens, para a razão MSPA/ MSR, apresentou valor estatisticamente superior em alta disponibilidade de nitrogênio (Tabela 1), demonstrando a maior proporção da massa seca de raízes sobre a massa seca da parte aérea, em baixa disponibilidade de nitrogênio.

$\mathrm{O}$ aumento da biomassa radicular ocorreu em todas as linhagens, em baixa disponibilidade de $\mathrm{N}$, induzindo a uma menor relação MSPA/ MSR dessas linhagens. Segundo Chun et al. (2005), a diminuição da relação MSPA/MSR sob estresse pode resultar de um crescimento reduzido da biomassa da parte aérea, com ou sem 
TABELA 1. Massa seca da parte aérea (MSPA), raiz (MSR), relação massa seca parte aérea/raiz (MSPA/MSR) e massa seca total (MSTO) de duas plantas por vaso, de seis linhagens de milho, em baixa $(\mathrm{N}-)$ e alta $(\mathrm{N}+)$ disponibilidade de nitrogênio ${ }^{1}$.

\begin{tabular}{ccccccccc}
\hline \multirow{2}{*}{ Linhagens } & \multicolumn{2}{c}{ MSPA(g) } & \multicolumn{2}{c}{ MSR(g) } & \multicolumn{2}{c}{ MSPA/MSR } & \multicolumn{2}{c}{ MSTO(g) } \\
\cline { 2 - 8 } & $\mathbf{N}-$ & $\mathbf{N}+$ & $\mathbf{N}-$ & $\mathbf{N}+$ & $\mathbf{N}-$ & $\mathbf{N}+$ & $\mathbf{N}-$ & $\mathbf{N}+$ \\
\hline CMS 28-7.1 & $2,02 \mathrm{a}$ & $3,01 \mathrm{ab}$ & $1,33 \mathrm{a}$ & $1,22 \mathrm{a}$ & $1,58 \mathrm{a}$ & $2,49 \mathrm{a}$ & $3,36 \mathrm{a}$ & $4,23 \mathrm{a}$ \\
CMS 28-8.1 & $2,09 \mathrm{a}$ & $3,36 \mathrm{a}$ & $1,22 \mathrm{a}$ & $1,16 \mathrm{a}$ & $1,82 \mathrm{a}$ & $2,91 \mathrm{a}$ & $3,31 \mathrm{a}$ & $4,53 \mathrm{a}$ \\
CMS 28-9.2 & $1,71 \mathrm{a}$ & $3,2 \mathrm{a}$ & $1,26 \mathrm{a}$ & $1,02 \mathrm{a}$ & $1,34 \mathrm{a}$ & $3,15 \mathrm{a}$ & $2,97 \mathrm{a}$ & $4,23 \mathrm{a}$ \\
CMS 28-10.2 & $1,67 \mathrm{a}$ & $3,11 \mathrm{a}$ & $1,47 \mathrm{a}$ & $0,96 \mathrm{a}$ & $1,2 \mathrm{a}$ & $3,33 \mathrm{a}$ & $3,14 \mathrm{a}$ & $4,07 \mathrm{a}$ \\
CMS 28-17.2 & $1,72 \mathrm{a}$ & $2,9 \mathrm{ab}$ & $1,28 \mathrm{a}$ & $1,06 \mathrm{a}$ & $1,41 \mathrm{a}$ & $2,72 \mathrm{a}$ & $3,00 \mathrm{a}$ & $3,97 \mathrm{ab}$ \\
CMS 28-19.1 & $2,02 \mathrm{a}$ & $1,98 \mathrm{~b}$ & $1,26 \mathrm{a}$ & $0,99 \mathrm{a}$ & $1,61 \mathrm{a}$ & $2,01 \mathrm{a}$ & $3,28 \mathrm{a}$ & $2,97 \mathrm{~b}$ \\
\hline Média Geral & $1,87 \mathrm{~B}$ & $2,93 \mathrm{~A}$ & $1,30 \mathrm{~A}$ & $1,07 \mathrm{~A}$ & $1,49 \mathrm{~B}$ & $2,77 \mathrm{~A}$ & $3,18 \mathrm{~B}$ & $4,00 \mathrm{~A}$ \\
\hline CV (\%) & 18,7 & 15,8 & 16,8 & 14,2 & 28,5 & 22,8 & 11,0 & 11,8 \\
\hline
\end{tabular}

${ }^{1}$ Médias seguidas pelas mesmas letras minúsculas, na vertical, e maiúsculas, na horizontal, para cada característica, não diferem significativamente pelo teste de Tukey, a 5\% de probabilidade.

aumento da biomassa radicular, podendo, assim, ser uma resposta adaptativa apresentada pela planta, maximizando a habilidade em adquirir uma quantidade maior de $\mathrm{N}$ do solo quando esse elemento se encontra em baixa disponibilidade.

Foram observados valores estatísticamente diferentes entre as linhagens para comprimento (CR), volume (VR) e superfície (SR) radicular, em baixa e alta disponibilidade de nitrogênio, com exceção do VR, em alta disponibilidade de $\mathrm{N}$ (Tabelas 2, 3 e 4). A média geral das seis linhagens, para SRMF e VRMF, foi estatisticamente maior em baixa disponibilidade de $\mathrm{N}$, sendo que, também nessa disponibilidade nutricional, houve a formação de quatro diferentes classes entre as médias do VRMF, com destaque para as linhagens CMS 28-7.1, CMS 28-8.1 e CMS 28-17.2.

A avaliação do VRMF sugere a presença de diferentes respostas da morfologia do sistema radicular das linhagens de milho cultivadas em ambientes contrastantes quanto à disponibilidade de nitrogênio. A quantificação de raízes finas com maior precisão, por meio da análise de características radiculares através do uso de imagens digitais, pode ser mais esclarecedora a respeito das respostas morfológicas do sistema radicular de linhagens de milho cultivadas sob estresse de nitrogênio. $\mathrm{O}$ uso de características radiculares em estudos de modelagem, para maior captura de nitrato, em plantas de lupim (Lupinus angustifolius), sugeriu o estudo 
TABELA 2. Comprimento de raiz muito fina (CRMF), comprimento de raiz fina (CRF), comprimento de raiz grossa (CRG), comprimento de raiz (CR) de duas plantas por vaso, de seis linhagens de milho, em baixa $(\mathrm{N}-)$ e alta $(\mathrm{N}+)$ disponibilidade de nitrogênio ${ }^{1}$.

\begin{tabular}{ccccccccc}
\hline \multirow{2}{*}{ Linhagens } & \multicolumn{2}{c}{ CRMF (cm) } & \multicolumn{2}{c}{ CRF (cm) } & \multicolumn{2}{c}{ CRG (cm) } & \multicolumn{2}{c}{ CR (cm) } \\
\cline { 2 - 8 } & $\mathbf{N}-$ & $\mathbf{N}+$ & $\mathbf{N}-$ & $\mathbf{N}+$ & $\mathbf{N}-$ & $\mathbf{N}+$ & $\mathbf{N}-$ & $\mathbf{N}+$ \\
\hline CMS 28-7.1 & $4342,45 \mathrm{ab}$ & $3635,64 \mathrm{abc}$ & $358,94 \mathrm{ab}$ & $284,60 \mathrm{a}$ & $119,74 \mathrm{a}$ & $69,60 \mathrm{a}$ & $4821,14 \mathrm{ab}$ & $3989,84 \mathrm{abc}$ \\
CMS 28-8.1 & $4815,86 \mathrm{a}$ & $4318,44 \mathrm{ab}$ & $364,92 \mathrm{a}$ & $326,34 \mathrm{a}$ & $103,54 \mathrm{a}$ & $87,48 \mathrm{a}$ & $5284,13 \mathrm{a}$ & $4732,26 \mathrm{ab}$ \\
CMS 28-9.2 & $4248,20 \mathrm{ab}$ & $4561,13 \mathrm{a}$ & $237,58 \mathrm{bc}$ & $287,53 \mathrm{a}$ & $59,20 \mathrm{a}$ & $86,08 \mathrm{a}$ & $4545,00 \mathrm{ab}$ & $4934,75 \mathrm{a}$ \\
CMS 28-10.2 & $3804,30 \mathrm{~b}$ & $3474,60 \mathrm{bc}$ & $232,33 \mathrm{c}$ & $280,28 \mathrm{a}$ & $48,96 \mathrm{a}$ & $78,09 \mathrm{a}$ & $4085,57 \mathrm{~b}$ & $3832,98 \mathrm{bc}$ \\
CMS 28-17.2 & $4529,11 \mathrm{ab}$ & $3169,09 \mathrm{c}$ & $343,89 \mathrm{abc}$ & $303,52 \mathrm{a}$ & $96,06 \mathrm{a}$ & $77,00 \mathrm{a}$ & $4969,71 \mathrm{ab}$ & $3549,55 \mathrm{c}$ \\
CMS 28-19.1 & $4152,36 \mathrm{ab}$ & $3308,04 \mathrm{c}$ & $286,11 \mathrm{abc}$ & $234,53 \mathrm{a}$ & $80,55 \mathrm{a}$ & $49,85 \mathrm{a}$ & $4519,01 \mathrm{ab}$ & $3592,43 \mathrm{c}$ \\
\hline Média Geral & $4315,38 \mathrm{~A}$ & $3744,49 \mathrm{~A}$ & $303,96 \mathrm{~A}$ & $286,13 \mathrm{~A}$ & $84,68 \mathrm{~A}$ & $74,69 \mathrm{~A}$ & $4704,03 \mathrm{~A}$ & $4105,31 \mathrm{~A}$ \\
\hline CV (\%) & 10,9 & 5,9 & 16,7 & 16,4 & 40,0 & 34,0 & 10,4 & 5,1 \\
\hline
\end{tabular}

${ }^{1}$ Médias seguidas pelas mesmas letras minúsculas, na vertical, e maiúsculas, na horizontal, para cada característica, não diferem significativamente pelo teste de Tukey, a 5\% de probabilidade.

TABELA 3. Volume de raízes muito finas (VRMF), volume de raízes finas (VRF), volume de raízes grossas (VRG), volume de raiz (VR) de duas plantas por vaso, de seis linhagens de milho, em baixa $(\mathrm{N}-)$ e alta $(\mathrm{N}+)$ disponibilidade de nitrogênio ${ }^{1}$.

\begin{tabular}{ccccccccc}
\hline \multirow{2}{*}{ Linhagens } & \multicolumn{2}{c}{ VRMF $\left(\mathbf{c m}^{3}\right)$} & \multicolumn{2}{c}{ VRF $\left(\mathbf{c m}^{3}\right)$} & \multicolumn{2}{c}{ VRG $\left(\mathbf{c m}^{3}\right)$} & \multicolumn{2}{c}{ VR $\left(\mathbf{c m}^{3}\right)$} \\
\cline { 2 - 8 } & $\mathbf{N}-$ & $\mathbf{N}+$ & $\mathbf{N}-$ & $\mathbf{N}+$ & $\mathbf{N}-$ & $\mathbf{N}+$ & $\mathbf{N}-$ & $\mathbf{N}+$ \\
\hline CMS 28-7.1 & $10,67 \mathrm{abc}$ & $8,27 \mathrm{ab}$ & $11,84 \mathrm{a}$ & $9,22 \mathrm{a}$ & $23,73 \mathrm{a}$ & $11,03 \mathrm{a}$ & $46,26 \mathrm{a}$ & $28,53 \mathrm{a}$ \\
CMS 28-8.1 & $11,56 \mathrm{a}$ & $9,75 \mathrm{a}$ & $11,90 \mathrm{a}$ & $10,64 \mathrm{a}$ & $19,08 \mathrm{a}$ & $16,66 \mathrm{a}$ & $42,54 \mathrm{ab}$ & $37,06 \mathrm{a}$ \\
CMS 28-9.2 & $8,51 \mathrm{~d}$ & $9,03 \mathrm{a}$ & $7,68 \mathrm{ab}$ & $9,33 \mathrm{a}$ & $9,39 \mathrm{a}$ & $15,83 \mathrm{a}$ & $25,59 \mathrm{~b}$ & $34,20 \mathrm{a}$ \\
CMS 28-10.2 & $8,85 \mathrm{~cd}$ & $8,88 \mathrm{ab}$ & $7,36 \mathrm{~b}$ & $9,09 \mathrm{a}$ & $7,11 \mathrm{a}$ & $14,13 \mathrm{a}$ & $23,36 \mathrm{~b}$ & $32,12 \mathrm{a}$ \\
CMS 28-17.2 & $10,99 \mathrm{ab}$ & $8,20 \mathrm{ab}$ & $11,19 \mathrm{ab}$ & $9,80 \mathrm{a}$ & $18,73 \mathrm{a}$ & $12,65 \mathrm{a}$ & $40,93 \mathrm{ab}$ & $30,66 \mathrm{a}$ \\
CMS 28-19.1 & $9,24 \mathrm{bcd}$ & $7,15 \mathrm{~b}$ & $9,24 \mathrm{ab}$ & $7,45 \mathrm{a}$ & $14,30 \mathrm{a}$ & $8,34 \mathrm{a}$ & $32,79 \mathrm{ab}$ & $22,94 \mathrm{a}$ \\
\hline Média Geral & $9,97 \mathrm{~A}$ & $8,55 \mathrm{~A}$ & $9,873 \mathrm{~A}$ & $9,25 \mathrm{~A}$ & $15,39 \mathrm{~A}$ & $13,11 \mathrm{~A}$ & $35,23 \mathrm{~A}$ & $30,92 \mathrm{~A}$ \\
\hline CV (\%) & 8,0 & 8,0 & 18,0 & 17,0 & 55,0 & 42,0 & 28,3 & 23,0 \\
\hline
\end{tabular}

${ }^{1}$ Médias seguidas pelas mesmas letras minúsculas, na vertical, e maiúsculas, na horizontal, para cada característica, não diferem significativamente, pelo teste Tukey, a 5\% de probabilidade 
do volume radicular como adequado para a melhor absorção do nitrato disponível (Dunbabin, 2002).

Sistemas radiculares de plantas podem responder a mudanças ambientais, como a baixa disponibilidade de nutrientes (Vamerali et al., 2003) ou água (Liu et al., 2005). Duas maneiras dessa plasticidade radicular são bem conhecidas, podendo ocorrer elevação local da cinética de absorção de nutrientes ou água ou o aumento da proliferação radicular (Robinson, 2001). Esses dois mecanismos podem exercer importante função na aquisição de nutrientes, produzindo algum grau de compensação, para desuniformidade da disponibilidade de nutrientes para a planta.

A inibição do crescimento de raízes laterais em altas concentrações de nitrato, no meio de cultivo, ocorre devido ao acúmulo de nitrato nas partes aéreas das plantas, fazendo com que haja uma inibição do fluxo de auxina para as raízes, prejudicando o crescimento de raízes laterais, pois a auxina é um importante hormônio que regula processos fisiológicos nas plantas, como o crescimento e o desenvolvimento radicular (Liu et al., 2006). A inibição do crescimento de raízes laterais, em plantas de milho submetidas a $20 \mathrm{mM}$ de nitrato, durante 12 dias de incubação, em solução nutritiva, foi devido à redução do alongamento celular na zona de alongamento radicular, como consequência da diminuição do transporte de auxina da parte aérea para a raiz, através do floema (Tian et al., 2008).

As linhagens CMS 28-7.1 e CMS 288.1 apresentaram médias superiores em todas

TABELA 4. Superfície de raízes muito finas (SRMF), superfície de raízes finas (SRF), superfície de raízes grossas (SRG), superfície de raiz (SR) de duas plantas por vaso, de seis linhagens de milho, em baixa $(\mathrm{N}-)$ e alta $(\mathrm{N}+)$ disponibilidade de nitrogênio ${ }^{1}$.

\begin{tabular}{ccccccccc}
\hline \multirow{2}{*}{ Linhagens } & \multicolumn{2}{c}{ SRMF $\left(\mathbf{c m}^{2}\right)$} & \multicolumn{2}{c}{ SRF $\left(\mathbf{c m}^{2}\right)$} & \multicolumn{2}{c}{ SRG $\left(\mathbf{c m}^{2}\right)$} & \multicolumn{2}{c}{ SR $\left(\mathbf{c m}^{2}\right)$} \\
\cline { 2 - 9 } & $\mathbf{N}-$ & $\mathbf{N}+$ & $\mathbf{N}-$ & $\mathbf{N}+$ & $\mathbf{N}-$ & $\mathbf{N}+$ & $\mathbf{N}-$ & $\mathbf{N}+$ \\
\hline CMS 28-7.1 & $610,65 \mathrm{abc}$ & $473,17 \mathrm{abc}$ & $226,47 \mathrm{a}$ & $178,02 \mathrm{a}$ & $428,87 \mathrm{a}$ & $293,68 \mathrm{a}$ & $1266 \mathrm{ab}$ & $944,87 \mathrm{ab}$ \\
CMS 28-8.1 & $670,17 \mathrm{a}$ & $564,00 \mathrm{a}$ & $228,98 \mathrm{a}$ & $204,84 \mathrm{a}$ & $425,13 \mathrm{ab}$ & $363,45 \mathrm{a}$ & $1324,29 \mathrm{a}$ & $1132,30 \mathrm{a}$ \\
CMS 28-9.2 & $523,25 \mathrm{bc}$ & $548,22 \mathrm{ab}$ & $148,46 \mathrm{ab}$ & $179,94 \mathrm{a}$ & $306,34 \mathrm{bc}$ & $360,90 \mathrm{a}$ & $978,06 \mathrm{c}$ & $1089,48 \mathrm{a}$ \\
CMS 28-10.2 & $513,33 \mathrm{c}$ & $485,51 \mathrm{abc}$ & $143,75 \mathrm{~b}$ & $175,40 \mathrm{a}$ & $277,96 \mathrm{c}$ & $308,57 \mathrm{a}$ & $935,04 \mathrm{c}$ & $969,48 \mathrm{ab}$ \\
CMS 28-17.2 & $624,60 \mathrm{ab}$ & $444,50 \mathrm{bc}$ & $215,53 \mathrm{ab}$ & $189,55 \mathrm{a}$ & $397,85 \mathrm{abc}$ & $288,61 \mathrm{a}$ & $1237,98 \mathrm{ab}$ & $922,73 \mathrm{ab}$ \\
CMS 28-19.1 & $543,30 \mathrm{bc}$ & $414,78 \mathrm{c}$ & $178,74 \mathrm{ab}$ & $145,27 \mathrm{a}$ & $342,99 \mathrm{abc}$ & $246,38 \mathrm{a}$ & $1065,03 \mathrm{bc}$ & $806,43 \mathrm{~b}$ \\
\hline Média Geral & $580,88 \mathrm{~A}$ & $488,38 \mathrm{~A}$ & $190,32 \mathrm{~A}$ & $178,83 \mathrm{~A}$ & $363,19 \mathrm{~A}$ & $310,26 \mathrm{~A}$ & $1134,4 \mathrm{~A}$ & $977,48 \mathrm{~A}$ \\
\hline CV (\%) & 8,4 & 6,1 & 17,6 & 17,5 & 14,5 & 13,1 & 9,5 & 8,5 \\
\hline
\end{tabular}

\footnotetext{
${ }^{1}$ Médias seguidas pelas mesmas letras minúsculas, na vertical, e maiúsculas, na horizontal, para cada característica, não diferem significativamente, pelo teste Tukey, a 5\% de probabilidade
} 


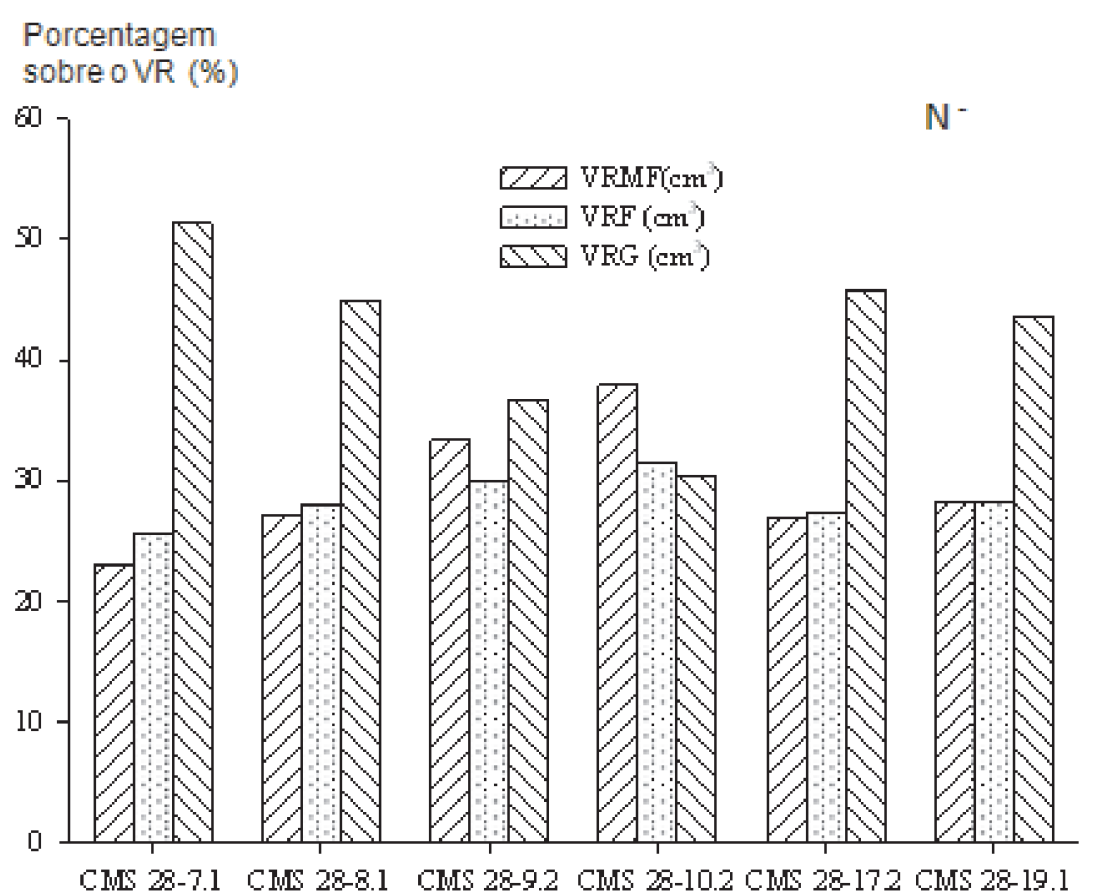

FIGURA 1. Proporção (\%) do volume de raízes muito finas (VRMF), volume de raízes finas (VRF) e volume de raízes grossas (VRG) em relação ao volume radicular (VR) de duas plantas por vaso, de seis linhagens de milho, avaliadas em baixa disponibilidade de nitrogênio $\left(\mathrm{N}^{-}\right)$no solo.

as características avaliadas, tanto em baixa quanto em alta disponibilidade de nitrogênio. Entretanto, a linhagem CMS 28-10.2 apresentou menor CRMF, CRF, CR, VRMF, VRF, VR, SRMF, SRF, SRG e SR do que outras linhagens, quando cultivadas em baixa disponibilidade de nitrogênio.

Em baixa e em alta disponibilidade de nitrogênio, houve diferença no percentual do VRMF, VRF e VRG, em relação ao VR, entre as linhagens contrastantes, na eficiência da utilização de nitrogênio
(Figuras 1 e 2, respectivamente). A linhagem eficiente na utilização de nitrogênio CMS 28-7.1 apresentou seu volume de raízes distribuído em 51,3\% de VRG, 25,6\% de VRF e 23,1\% de VRMF, ao contrário da linhagem ineficiente CMS 28-10.2, que apresentou $37,9 \%$ de VRMF, 31,5\% de VRF e $30,4 \%$ de VRG, sugerindo que a eficiência na utilização de nitrogênio por linhagens de milho poderia estar relacionada à presença de um volume de raízes muito finas eficientes na absorção de nitrogênio do solo. 


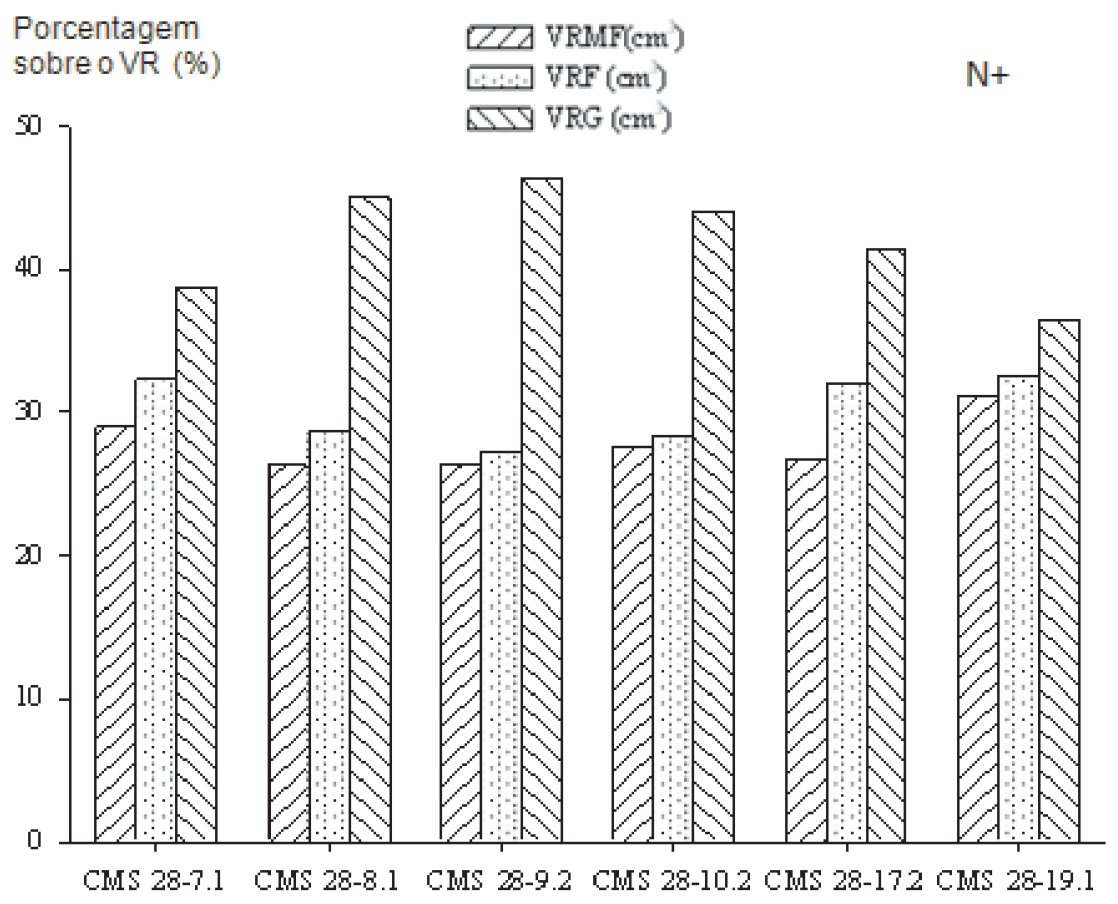

FIGURA 2. Proporção (\%) do volume de raízes muito finas (VRMF), volume de raízes finas (VRF) e volume de raízes grossas (VRG) em relação ao volume radicular (VR) de duas plantas por vaso, de seis linhagens de milho, avaliadas em alta disponibilidade de nitrogênio $(\mathrm{N}+)$ no solo.

\section{Literatura Citada}

BANZIGER, M.; EDMEADES, G. O.; BECK, E. D.; BELLON, M. Breeding for drought and nitrogen stress tolerance in maize from theory to practice. Mexico, D.F: CIMMYT, $2000.68 \mathrm{p}$.

BÄNZIGER, M.; EDMEADES G. O.; LAFITTE, H. R. Physiological mechanisms contributing to the increased $\mathrm{N}$ stress tolerance of tropical maize selected for drought tolerance. Field Crops Research, Amsterdam, v. 75, p. 223-233, 2002.
BOUMA, T. J.; NIELSON, K. L.; KOUTSAAL, B. Sample preparations and scanning protocol for computerized analyses of root length and

diameter. Plant and Soil, The Hague, v. 218, p.185-196, 2000.

BHOM, W. Methods of studying root systems. New York: Springer-Verlag, 1979.189 p.

CANTÃO, F. R. O. Marcadores morfológicos de raiz em genótipos de milho contrastantes para tolerância à seca em resposta a estresses 
de fósforo e alumínio. 2007. 98 f. Dissertação (Mestrado em Fisiologia Vegetal) - Universidade Federal de Lavras, Lavras.

COSTA, C.; DWYER, L. M.; ZHOU, X.; DUTILlEUL, P.; HAMEL, C.; REID, L. M.; SMITH, D. L. Root Morphology of Contrasting Maize Genotypes. Agronomy Journal, Madison, v. 94, p. 96-101, 2002.

CRUZ, C. D. Programa GENES: aplicativo computacional em genética e estatística versão Windows. Viçosa, MG: UFV, 2001. 442 p.

CHUN, L.; MI, G.; LI, J.; CHEN, F.; ZHANG, F. Genetic analysis of maize root characteristics in response to low nitrogen stress Plant and Soil, The Hague, v. 276, p.369-382, 2005.

DEUNER, S.; NASCIMENTO, R.; FERREIRA, L. S.; BADINELLI, P. G.; KERBER, R. S. A. Adubação foliar e via solo nitrogênio em plantas de milho em fase inical de desenvolvimento. Ciência e Agrotecnologia, Lavras, v. 32, n. 5, p. 1359-1365, 2008.

DUNBABIN, V. M. The effect of root system form and function on nitrate uptake by lupins in a leaching environment. 2002. Tese (Doutorado) - University of Western Australia, Perth.

EDMEADES, G. O.; BOLANOS J.; BÄNZIGER M.; RIBAUT J. M.; WHITE, J. W; REYNOLDS, M. P.; LAFITTE, H. R. Improving crop yields under water deficits in the tropics. In: INTERNATIONAL CROP SCIENCE CONGRESS, 2., 1996, New Delhi. Crop productivity and sustainability - shaping the future: proceedings. Enfield: Science Publishers, 1998. p. 437-451. Editado por V. L. Chopra, R. B. Singh e A. Varma.

FIDELIS, R. R.; MIRANDA, G. V.; SANTOS, I. C.; GAlVÃO, J. C. C.; PELUZIO, J. M.; LIMA, S. O. Fontes de germoplasma de milho para estresse de baixo nitrogênio. Pesquisa Agropecuária Tropical, Goiânia, v. 37, n. 3, p.147-153, 2007.

GALLAIS, A.; HIREL, B. An approach to the genetics of nitrogen use efficiency in maize. Journal of Experimental Botany, London, v. 55, n. 396, p. 295-306, 2004.

GALLAIS, A.; COQUE, M.; Genetic variation and selection for nitrogen use efficiency in maize: a synthesis. Maydica, Bergamo, v. 50, p. 531-537, 2005.

HODGEN, P. J.; FERGUSON, R. B.; SHANAHAN, J. F.; SCHEPERS, J. S. Uptake of Point Source Depleted 15N Fertilizer by Neighboring Corn Plants. Agronomy Journal, Madison, v. 101, p. 99-105, 2009.

LIU, F.; JENSEN, C. R.; ANDERSEN, M. $\mathrm{N}$. A review of drought adaptation in crop plants: changes in vegetative and reproductive physiology induced by ABA-based chemical signals. Australian Journal of Agricultural Research, Victoria, v. 56, p.1245-1252, 2005.

LIU, P. W.; IVANOV, I. I.; FILLEUR, S.; GAN, Y.; REMANS, T.; FORDE, B. G. Review 
Nitrogen Regulation of Root Branching. Annals of Botany, London, v. 97, p. 875-881, 2006.

MIRANDA, G. V.; GODOY, C. L.; GALVÃO, J. C. C.; SANTOS, I. C.; ECKERT, F. R.; SOUZA, L. V. Selection of discrepant maize genotypes for nitrogen use efficiency by a chlorophyll meter. Crop Breeding and Applied Biotechnology, Londrina, v. 5, p. 451-459, 2005.

O’NEILL， P. M.; SHANAHAN， J. F.; SCHEPERS, J. S. Use of chlorophyll fluorescence assessments to differentiate corn hybrid response to variable water conditions. Crop Science, Madison, v. 46, p. 681-687, 2006.

PRESTERL, T.; SEITZ, G.; LANDBECK, M.; THIEMT, W.; SCHMIDT, W.; GEIGER, H. H. Improving nitrogen use efficiency in European maize: estimation of quantitative parameters. Crop Science, Madison, v. 43, p. 1259-1265, 2003.

PURCINO, A. A. C.; LIMA, T. R.; PINTO, A. C.; CARNEIRO, N. P.; SOUZA, I. R. P de; MARRIEL, I. E.; PARENTONI, S. N.; DURAES, F.; CARVALHO, I. Glutamine synthetase response to nitrate in maize genotypes of contrasting nitrogen use efficiency. Maydica, Bergamo, v. 53, p.101-109, 2008.

ROBERTS, D. F., ADAMCHUK, V. I.; SHANAHAN, J. F.; FERGUSON, R. B.; SCHEPERS, J. S. Optimization of Crop Canopy
Sensor Placement for Measuring Nitrogen Status in Corn. Agronomy Journal, Madison, v. 101, p. 140-149, 2009.

ROBINSON, D. Root proliferation, nitrate inflow and their carbon costs during nitrogen capture by competing plants in patchy soil. Plant and Soil, The Hague, v. 232, p. 41-50, 2001.

RUBIO, G.; LYNCH. J. P. Compensation among root classes in Phaseolus vulgaris L. Plant and Soil, The Hague, v. 290, p. 307-321, 2007.

SOUZA, L. V.; MIRANDA, G. V.; GALVÃO, J. C. C.; ECKERT, F. R.; MANTOVANI, E. E.; LIMA, R. O.; GUIMARÃES, L. J. M. Genetic control of grain yield and nitrogen use efficiency in tropical maize Pesquisa Agropecuária Brasileira, Brasília, DF, v. 43, p.1517-1523, 2008.

TIAN, Q.; CHEN, F.; LIU, J.; ZHANG, F.; MI, G. Inhibition of maize root growth by high nitrate supply is correlated with reduced IAA levels in roots. Journal of Plant Physiology, Stuttgart, v. 165, p. 942-951, 2008.

VAMERALI, T.; SACCOMANI, M.; BONA, S.; MOSCA, G.; GUARISE, M.; GANIS. A. A comparison of root characteristics in relation to nutrient and water stress in two maize hybrids. Plant and Soil, The Hgue, v. 255, p.157-167, 2003. 
